\title{
Lexico-Cultural Variations in Product Naming: A Note on the Names of Handcrafted Soaps
}

\author{
LINDSEY N. H. ChEN \\ National Taiwain Normal University, Taipei, Taiwan
}

This comparative study examines the names of handcrafted soaps, a group of natural products that has become popular among environmentally conscientious consumers in recent years. In particular, 339 names of US-based handcrafted soaps (US-HS) and 324 names of Taiwan-based shou gong fei zhou 'lit. hand craft soap' (TW-HS) were analyzed and compared. The findings show that plant and food names predominate in HS names and reflect the cultural and herbal preferences in both regions. In addition, colorful, allusive, and novel coinages were found to be frequent among US-HS names. By comparison, function-denoting words were mainly found among TW-HS names. This study provides insights into potential cross-cultural variations in consumer product naming.

KEYWORDS product names, soap, plant names, phytonymy, Asia

\section{Introduction}

Many recent onomastic studies have focused on product naming (see Danesi 20I I; Pérez Hernández 20I3; Nuessel 20I8). Altogether, the literature has demonstrated the crucial role that names play in the marketing and advertisement of consumer products. Through creative language and strategies, companies can paint an image of a product that increases its desirability. Coinages of product names may be classified as descriptive, suggestive, iconic, or symbolic (Danesi 20II). A product name, then, is not just a printed title: the word or words are used to identify a company, service, or concept. Furthermore, if the name is evaluated positively or has positive connotation in the consumers' view, the product stands to succeed in the home market and perhaps even in foreign markets. Onomastic research thus has enriched our understanding not only of consumer 
products names specifically, but also the art and science of product naming in general. Haig (20II), for instance, suggested some important characteristics for product names-crucially, a name should be short, unique, distinctive, easily pronounced, and easily translated into foreign languages. Focusing on US products in the Chinese culture as a case study, Dong and Helms (200I) analyzed patterns of brand name translation and showed that appropriate names make a significant difference in the successful introduction of new brands in the US as well as abroad.

More recently, Chen (2019) examined the English names of handcrafted soaps (HS), a group of natural products that has become popular among environmentally conscientious consumers in recent years. Here, the term 'HS product names' is not synonymous with 'HS brand names' but rather, refers to names of specific products subsumed under a particular HS brand. For example, the USHS brand Soaptopia has the following named products in its "Bar Soap" line: Lemon Rosemary, Crème Vanilla, Pepperlyptus Rex and Love Thyme All the Time. Chen 2019 uncovered several linguistic strategies in English used to formulate HS names. These included vowel lengthening, semi-syllable break, repetition, homophonous substitution, numeral in place of word, imperative, and code-switching. The study also showed how names helped re-frame traditional soaps and soap-making as hip and trendy. It also provided general insights into the naming conventions of natural products in North America.

The present study builds on the previous research by investigating the potential lexico-cultural variations in the naming of HS products. While different retail strategies to market "eco-friendly" products have been discussed before (see Hall 2008; Ottman 20II; Sarkar 20I2), few studies have compared names of American and Asian consumer products from a lexico-cultural perspective. In this particular investigation, 339 names of US-based handcrafted soaps (US-HS) and 324 names of Taiwan-based shou gong fei zhou 'lit. hand craft soaps' (TW-HS) were analyzed and compared. The findings show that HS names commonly incorporate plant names and descriptors that may be used as a marketing strategy to appeal to consumers. Whereas colorful, allusive, and unconventional coinages were found to be frequent among US-HS names, function- denoting words were mainly found among TW-HS names. In addition, the influence of cultural tradition and herbal preferences on the naming of natural products was observed.

\section{On Handcrafted Soaps}

Handcrafted soaps are handmade skincare soaps that contain no artificial and synthetic ingredients. They are environment-friendly because these leave no toxic residues. Unlike commercial soaps, handcrafted soaps are produced in small batches from a process called "saponification" which involves creating a mixture of fat, oil, and lye (Cross 2015). For extra dermatological benefits, essential oils, such as anti- bacterial tea tree oil or refreshing peppermint oil, are added. For instance, the list of ingredients for the Lavender Lime soap from Parsonage 
Soap Company is simply "Saponified oil of palm, coconut, olive, lavender and lime essential oils” (PSC 2015).

\section{Methodology}

For this study, 324 names of HS products from I 2 Taiwanese brands were collected and analyzed. The twelve brands (in English, as provided on the promotional brochures) were Ann Chen, Ba Di Luo Si, Claire's, Emma Flora, Eversun, Loveherb, Monga, Soapmore, Tea Soap, Wen-Shan, Yuan, and Yusoap. For the TW-HS dataset, the examples in traditional Chinese characters were transcribed using the Hanyu Pinyin romanization system and translation was provided. For example, ai cao kang jun (lit. 'Mugwort Anti-bacteria') is a name that contains a common plant name ai cao 'mugwort' and the term kang jun 'anti-bacteria.' In Chinese, the same word or lexical item can be used for different parts of speech. For instance, the color term hong 'red' in HS names hong ju 'Red Yeast Rice' can be said to be clearly "adjectival." However, the modifier jing xin in the HS name jing xin kui mu could be translated to literally 'clear heart' or simply 'calming' (kui mu = 'cypress'). In such cases, the more felicitous English translation - or what was judged to be the more natural expression for marketing purposes - is presented (e.g., 'Calming Cypress').

Different content words were then identified and tabulated in a spreadsheet, as some lexical items have multiple occurrences. For organizational purposes, HS names were placed under the feature categories Plant Names (P), Names of Food/Beverages (FnB), Linguistic Wordplay (W), and Descriptive Names (D), if applicable. The last category could also include place names or function-denoting terms. For example, “Anti-bacterial Mugwort" would have been assigned the features $\mathrm{P}$ and $\mathrm{D}$.

For the cross-cultural comparison, the dataset containing 339 US-HS names were based on those examined in Chen (2019). The HS names in English were obtained from the main websites of nine US-based makers of natural handcrafted soaps. These nine brands were A Wild Soap Bar, Chagrin Valley Soap \& Salve, Kalliste Soap Shop, Jennifer's Handmade Soap, SLAB, STEM, Soaptopia, Sweet Cheeks Soap, and The Parsonage Soap Company. The examples were organized similarly. Finally, published and online references were consulted to verify information on plants, essential oils, and herbal remedies.

\section{Results ${ }^{1}$}

\section{Plant Names}

Here, plant names include names of flowers and fruits. Overall, the incorporation of plant names predominate, accounting for $60 \%$ and $80 \%$ of the samples in the US-HS (202) and TW-HS (258) names lists, respectively. In general, these examples $^{\mathrm{I}}$ reflect the diversity within the botanical world, highlighting the versatility of plant species. Names of florals found in both lists include 'calendula' 
(jin kui), 'chamomile' (yang gan ju), 'honeysuckle' (jin yin hua), 'jasmine' (mo li), 'lavender' (xun yi cao), 'magnolia' (yu lan hua), 'rose' (mei gui), and 'verbena' (ma bian cao). Names of fruits include 'apple' (ping guo), 'avocado' (lao li), 'coconut' (ye zi), 'lemon' (ning mun), 'orange/citrus' (cheng/gan ju), and 'peach/apricot' (tao). For names of culinary herbs, examples include 'ginger' (jiang), 'lemongrass' (xiang mao), 'mint' (bo he), 'rosemary' (mi die xiang), and 'thyme' (bai li xiang). For lighter veggie scents, 'carrot' ( $h u$ luo bo), 'cucumber' (buang gua), and 'loofah' (si gua) may be the keywords to search. HS names that include 'cedar' (xue song), eucalyptus (you jia li), 'frankincense' (ru xiang), 'myrrh' (mei yao), 'pine' (song mu), and 'sandalwood' (tan xiang) suggest woodsy and earthy aromas. Finally, four ingredient names often found in household products are 'aloe' (lu hui), 'oat' (mai), 'olive' (gan lan), 'shea' (ru mu), and 'tea tree' (cha shu). Tea tree, in particular, had widespread appeal, occurring 6 and 7 times in the US- and TW-HS lists, respectively. Its essential oil is known to have a fresh camphoraceous odor that can serve multiple purposes because of its disinfectant quality (Fox 2005). Additional examples appearing in both HS names lists are provided in Appendix I.

There were some slight differences in terms of frequency and variation of occurrence. A notable case is 'calendula' (jin kui), which appears once in the USHS list but $\mathrm{I} 2$ times in the TW-HS list. However, minty flavors (bo be 'mint') were identified 6 times in the TW list but I I times in the US list. Judging from the various names, berries appear to be more popular in North America. The following names were uncovered in the US- but not TW-HS list: bayberry, blackberry, blueberry, cranberry, huckleberry, juniper berry, raspberry, seaberry, and strawberry. While the US-HS list offered simply 'orange' or 'citrus,' TW-HS list contained a wide range of citrus-type fruits, including fo shou gan 'bergamot,' gan ju 'citrus,' jin ju 'kumquat,' $k u$ cheng 'bitter orange,' and tian ju 'sweet citrus.' Related to this finding, a similar type/token distinction was observed with references to 'salt.' The mineral name 'salt' (yen) occurred in both lists, but variations of this were more common in the TW-HS list: shi yan (Rock Salt), mei gui yan (Rose Salt), hai yan (Sea Salt), huo shan ni yan (Volcanic Mud Salt), and tai ping yang shen hai yan (Deep Pacific Salt).

Another contrast between the two regions was the number of names of exotic medicinal herbs in the TW-HS names list. Traditional Chinese Medicine (TCM) has existed for centuries as an internal and external treatment for medical conditions. Among them, zi cao 'gromwell' and ai ye 'mugwort' were particularly popular, both appearing Io times. Collocated 4 times, hui ren 'pearl barley' and lu dou 'mung bean' both refer to active ingredients that are said to improve the condition of skin pigmentation and spots. Other names of TCM herbs are provided in Appendix 2. Notably, the onomastic samples show that various botanical parts can be put to use for commercial purposes. These parts are explicitly identified by words such as cao 'grass,' geng 'root,' guo 'fruit,' hua 'flower,' jeng 'needle,' mu 'wood,' $z i$ 'seed,' and ye 'leaf.' 


\section{Names of Food and Beverages}

In addition to the edible vegetables listed above, names of (other) food and beverages were also identified. Included in the US-HS names list were butter, chocolate, cinnamon, coffee, jam, yogurt, vanilla, (hot) cocoa, oatmeal cookie, and ginger snap-the names of which might be associated with warm, sweet aromas. Milk, a common breakfast staple in the USA, was referenced 6 times, two of which specifically designate Goat Milk. In particular, honey and oatmeal appeared I 5 and I4 times, respectively. These high figures attest to the popularity of these two ingredients, perhaps because of their moisturizing and soothing quality.

Many of the aforementioned food names also appeared in TW-HS names list, e.g., $k a$ fei 'coffee,' ke ke 'cocoa,' qiao ke li 'chocolate,' mi 'honey,' niu nai 'milk,' nai you 'butter' (lit. “milk oil”), yan mai 'oatmeal,' and you ge 'yogurt'. However, products like milk, yogurt, hot cocoa, and macchiato (ma chi duo) are not generally considered part of a traditional Asian diet. From a linguistic point of view, some of the phonetic renderings (e.g., $k a f e i>$ 'coffee', you ge > 'yogurt') may be evidence of lexical borrowing from English due to contact with western cultures. As Haugen (2009) pointed out, with the introduction of new plants and food preparation (i.e., "food borrowings") have come new lexical borrowings.

Handcrafted soap names also highlighted differences in beverage preference across cultures. As the HS names showed tea is more commonly consumed in the East. While 'Grapefruit Tea' and 'Sweet Tea' were found in the US-HS list, names of tea-infused HS in the TW list were more numerous, including $l u c h a$ (Green Tea, 3 instances), ku cha (Bitter Tea, 2 instances), luo shen hua cha (Roselle Tea), Sanxia mi xiang hong cha (Sanxia Honey Fragrant Red Tea)' Bi Luo Chun (type of green tea), and ri ben mo cha (Japanese Matcha Tea). The diverse assortment of tea identified is not surprising, as tea has a prominent place in Chinese culture. As an added benefit, these soaps could be used as a homemade remedy to treat sunburn skin and acne. By contrast, the US-HS list contained more names of cocktail-type drinks and appeared in examples such as Apple Jack, Farmbouse Cider, Beer, Honey Beer, Irish Tweed Beer, Sweet Moscato, Tequila Sunrise, Orange Ginger Spritzer, and Cocktails by the Pool. The only names of alcoholic beverages uncovered in the TW list were tai wan pi jou 'Taiwan Beer' and sake, a type of Japanese rice wine.

\section{Descriptive Names}

\section{Colorful Expressions}

References to faraway, exotic places were found among the colorful names in the US-HS list. These names, as originally noted in [Chen (2019)], include Arabian Spice, Egyptian Dragon, Java Spice, Moroccan Garden, Scottish Wedding, Shenandoah Woods, Tuscany Evening, Venice Vanyasa, Waterford Faire, Jamaica Me Crazy, Oceania, Thai on One, Weekend in Maui, and Gold Coast with African Black. Nature-inspired HS names are exemplified by Ocean 
Breeze, Blue Ridge Breezes, Sun Kissed, and Woodland Trail. The name Dead Sea Spa (and simply Spa) suggests a rich, luxurious experience that can rejuvenate the mind and body. Other descriptive names that can evoke dreamlike, magical and peaceful scenes are Moon Magic Natural, La La Lavender, Christmas Memories, Jazzy Blue, Shenandoah Starlight, Purple Haze, and Sweet Dreamweaver. HS names with amorous overtones are Earl's Girls, Red Hot Lover, Spread the Love, Sunny Baby, Sweet Bouquet, Victorian Rose, and Winter Serenade.

Place names were rare in the TW-HS list: only two were uncovered-Marseille and Sanxia. Marseille (6 occurrences) refers to the region in France renowned for its lavender variety. There were few descriptive names connoting love (dong zhi lian 'Winter Romance,' nong qing qiao ke li 'Passionate Chocolate,' mei gui guo zhi lian 'Romance of the Rosehip,' ai lian shan cha hua xi fa 'Loving Camellia Hairwash') or well-being (xing fu dou jiang 'Blessed Soy Milk,' yang sheng kui mu 'Healthy Living Cypress'). Other than these examples, descriptive TW-HS names were found to be more "practical" than colorful.

\section{Function-Denoting Terms}

Of the 324 TW-HS names, I 26 (39\%) contained terms denoting a single or multiple functions. For space reason, repeating modifiers are designated by $*$ in the examples given. Where the asterisk appears is where the modifying expression occurred.

As soaps are meant to clean away dirt, naturally, it was not surprising that words such as 'cleansing,' 'clarifying,' or 'purifying' were found. Among TW-HS names, 24 names tied to this concept were identified in various forms. Some examples were chun jing 'pure clean' (* gan lan 'Cleansing Olive'), jing shen 'clean body' (* ai cao 'Cleansing Mugwort'), jing fu 'clean skin' (tan xiang * 'Cleansing Sandalwood'), jing rou 'clean soft' (yang gan ju* 'Gentle Cleansing Chamomile'), jie jing 'clean clean' (cha su* 'Purifying Tea Tree'), jie fu 'clean skin' (zi dan * 'Cleansing Tournefortia montana'), jie yan 'clean face' (yi lan* 'Cleansing Ylangylang'), and qing $j i$ 'clear flesh' (* hui ren 'Clarifying Pearl Barley').

Handcrafted soaps do more than clean away dirt, however. Sometimes, they function as lotions and creams-helping to nourish, moisturize, and soften the skin. HS names incorporating such terms occurred 17 times. The function-denoting modifiers included zi yang 'nourish grow' (tian xin luo $l i *$ 'Nourishing Sweet Almond \& Avocado'), zi ruan 'nourish moisten' (ma sai xun yi cao * 'Nourishing Marseille Lavender'), rou fu 'soft skin' (* zhen guo 'Softening Hazelnut), ruan fu 'moisten skin' (zhen guo feng $m i *$ 'Moisturizing Hazelnut Honey'), and rou ruan 'soft moisten' (mei gui hong ni * 'Softening Rose \& Red Mud').

Certain scents are believed to calm and soothe one's mood. Three examples that fell under the category of 'calming/soothing' were jing xin 'clear heart' (* kui mu 'Calming Cypress'), tian jing 'tranquilizing' (* xue song 'Tranquilizing Cedar'), shu huan 'soothing' (* ke ke 'Soothing Cocoa,' * jing kui hua 'Soothing Calendula'), and shu ming 'allergy-relieving' (yan mai * 'Allergy-relieving Oat'). 
On a related note, the term ping an 'peace' appeared in seven instances, including ai cao * 'Peaceful Mugwort' and luo $l i$ * 'Peaceful Avocado.'

Certain scents are also thought to perk up one's mood. Thirteen terms in Chinese that were associated with the idea of 'energizing' and 'refreshing' were buo $l i$ 'alive power' (* shan ku gua 'Invigorating Bitter Melon'), xing fu 'awake face' (xiang mao ai cao * 'Awakening Lemongrass \& Mugwort'), huan cai 'brilliant color' (* guo cu 'Fruit Vinegar for Radiant Complexion'), qing liang 'light cool' (bo be * 'Refreshing Mint'), and qing shuang 'light refreshing' (bo be lu bui ${ }^{*}$ 'Refreshing Mint \& Aloe').

TW-HS that promote more advanced cosmetic treatment were also identified. The products' promise to exfoliate, firm, or even repair the skin may be particularly appealing to women over a certain age. Related examples were qu jiao zhi 'away flakiness' (xing tao be ren * 'Exfoliating Apricot Kernel'), jin zhi 'tight fine' (* si bo 'Firming Cypress,' hai tai * neng fu 'Seaweed for Tight Delicate Skin'), and xiu fu 'repair skin' ( $y$ u lan* 'Repairing Magnolia').

Notably, the occurrences of the [adj. + bai 'white'] compound may point to a cultural attitude about beauty that is pervasive in East Asian societies: the longstanding preference for light skin. This culture-specific phenomenon-or rather, according to critics, the dark side of the beauty industry-is revealed in the popularity of skin-whitening cosmetic products that have soared in recent years (Rondilla and Spickard 2007). Soapmakers, too, seem to be aware of this trend, and have promoted their products with names like jing bai yu long (Pure White Beauty), mei bai zhen zhu (Whitening Pearl), mei gui guo neng bai (Rosehip for Delicate White Skin) and hui ren lu dou huan bai (Pearl Barley Mung Bean for Bright White Skin).

The purported practical uses of HS were conveyed in other ways as well. Handcrafted soaps formulated specifically for babies (bao bei) were found in mo $\mathrm{cao}^{*}$ 'Desmodium caudatum for Baby' and ai cao * rou $\mathrm{fu}$ 'Mugwort for Baby Soft Skin.' For the sole purpose of face washing, HS with the designation xi yen 'face wash' (xiao huang gua * 'Cucumber Face Wash' and mei ren ning meng * 'Lemon Beauty Face Wash') were located. HS for pet care was suggested in xing wan chong wu 'Happy Pet'. Handcrafted soaps are also often sold as alternatives to shampoo, as evidenced by 23 instances of the term xi fa (lit. 'wash hair') among HS names. Plant names associated with hair washing included bai $l i$ xiang 'thyme,' dong gui 'Angelica sinensis,' fo shou gan 'bergamot,' jiang 'ginger,' mi die xiang 'rosemary,' and wu huan zhi 'soapberry.' Notably, the name of the traditional Chinese herb he shou wu 'Polygonum multiflorum' appeared five times. This herb is alleged to stimulate hair growth and even turn white hair to black (e.g., he shou wu liang he xi fa 'Polygonum multiflorum for Shiny Black Hair'). Finally, there was a keyword associated with household cleansers: jia shi 'housework' (e.g., ye you * 'Coconut Oil for Housework' and gan julcha shulka fei ${ }^{*}$ ching jie 'Citrus/Tea Tree/Coffee for House Cleaning').

Finally, TW-HS names with the prefix kang 'anti-' or the term fang 'prevent' suggested active fighting ingredients. Five names with this character morpheme 
included zi cao kang jun (Anti-bacterial Mugwort), kang dou huang lian (Antiacne Coptic chinensis), kang lao luo li (Age-defying Avocado), and tian ran xiang mao fang wen (Natural Lemongrass for Repelling Mosquitos). Two examples of oil-controlling HS were found: cha shu ka fei kang you (Oil-controlling Tea Tree \& Coffee) and zuo shou xiang kang you (Oil-controlling Coleus amboinicus).

Conversely, there are few lexical combinations in the US-HS names list that suggested what the key ingredient could achieve in terms of specific skincare benefits. The six identified on the list included three that were said to also benefit the hair (Shampoo, Yucca Root Shampoo \& Body, Juniper Berry Shampoo of Body); three specially formulated for gardeners (Gardeners, Gardener's Scrub, Gardener's Revenge); one for a common skin problem (Black Willow Bark for Acne), and the generically plain Shaving.

\section{Popular Culture Allusions/Cultural Symbols}

References to popular culture combined with clever wordplay were identified among US-HS names, as documented in Chen (2019). Allusive names appear to have been inspired by movies (Tequila Sunrise, Ocean's I2), television dramas (Miami Vice, Boardwalk Hempire), a cartoon character (Woody Wood Pepper), a creature of the Lost World (Pepperlyptus Rex), a fictional monster (Rozilla vs. Dry Skinea), bestselling novel (Soap Pray Love), a teen novel series (Sweet Bali High), a literary classic (Grapefruit Gatsby), a fairy tale (Beauty and the Beach), a music festival (Woodstock Revival), bands (Blue Pearl Jam, Coal Play), and a song title (Like a Virgin). Even song lyrics served as inspiration for HS names: Cleaning Me Softly with this Soap (song $\rightarrow$ soap, Roberta Flack) and I Can Think Clearly Now the Pain is Gone (rain $\rightarrow$ pain, Johnny Nash).

As the Chinese language has a very different orthographic system than US American English, the kinds of linguistic strategies or clever wordplay seen in US-HS names were not possible in TW-HS naming practices. Also, none of the TW-HS names alluded to (Chinese) pop culture. However, they did reveal preferences and facets that are meaningful to the Chinese culture. For example, names of florals highly symbolic in the Chinese culture were found: $j u$ bua 'chrysanthemum,' lian hua 'lotus,' gui hua 'osmanthus,' and yin bua 'cherry blossom.' These flowers are native to the Far East and frequently appear in works of art and poems. While the cherry blossom symbolizes the ephemeral nature, the chrysanthemum represents nobility and elegance. The lotus is a flower equated with the holy seat of Buddha. These were not the only culturally-relevant examples found. For centuries, sandalwood (tan xiang) has been used to make incense sticks for worshipping. A reference to natural indigo, ching dai, has been used as a main ingredient for dyed clothes in China for more than Iooo years. Indigenous to the Philippines, yi lan 'ylang-ylang' symbolizes femininity and is used extensively in perfumes. The reduplicative name is derived from the Tagalog term ilang-ilan, meaning "just a few, rare”. 


\section{Discussion}

Generally, the goal of product naming is to create a name that is easy to remember and is associated with a product feature. Charmasson (I988) stated that a product name should be (I) easy to pronounce and pleasant to the ear; and (2) morphologically short and simple. For example, an ideal and relevant name for a sport supplement product would be one associated with the idea or image of strength and energy e.g. Sculptress and FuelMax (GlaxoSmithKline). The car model name Odyssey, used the Japanese car-maker Honda Motor Company, is a fine name for an automobile as it conveys a sense of endurance and greatness. Names of handcrafted soaps, however, seem to defy the usual conventions and practices for product naming. Overall, HS names were found to be more informative than memorable or unique. Considering that plant names are incorporated into most of the samples, the naming practices of US- and TW-HS we not vastly different in this investigation. The appeal of many plants such as lavender, mint, olive, rose, and citrus seems universal. As the HS names illustrated, Mother Nature provides all humankind with wide diversity of plants that are roundly believed to be beneficial to people's dermatological and emotional well-being. Through HS names, consumers come to discover the unique versatility and potential healing power of plant species involved, some of which they probably have never even heard of. Whether in English or Chinese, HS names may simply but effectively project a message of health as they make a positive link to the "all-natural" feature that characterizes handcrafted soap products in general.

The references to plants in TW-HS names also found to be culturally informative. Around the world, plants are featured in many aspects of culture, including language, history, art, religion, and medicine. Through various examples, Kakudidi (2004) and Hsu and Harris (2010) showed how the cultural significance of plants could be gleaned from anthropological, ethnobotanical, and linguistic studies. Collectively, TW-HS names reflect the age-old tradition of TCM that is still widely accepted today. For more than 2500 years, TCM has been the dominant healing system and has often been regarded as the appropriate mode of treatment by specialists and patients and the Chinese have continued to utilize many indigenous herbs to treat ailments and cure diseases. In a way, the substantial number and diversity of the exotic plant names identified among the HS names affirms the general acceptance of traditional medicine within Asian societies even in the new millennium.

As shown in this investigation, the TW-HS names included various functiondenoting terms and keywords that direct consumers to specially-formulated HS for specific purposes. By comparison, the US-HS names liberally adopted colorful and allusive coinages. Both practices may be seen as a marketing strategy to appeal to consumers. Terms of utility further emphasize the value of handcrafted soaps. Though the benefits and advantages of certain botanicals have been widely studied and well-documented, the claims made in some of the HS names (e.g., "repairing," "invigorating," "whitening," "baby soft," "for shiny black 
hair") should be taken with a grain of salt. Nevertheless, colorful and witty names present a style that is potentially emotionally engaging and eye-catching to consumers. The associations with particular geographical locations may have extra appeal: using these soaps will transport users to an exotic tropical island or faraway land. Altogether, these names may paint an attractive picture in the minds of the buyers. They may help sell adventure, dreams, and even romance. In short, despite their exaggerated claims or colorful expressions, these words have the power to influence buyers in their purchasing decisions. As the anthropologist Laura Miller observed in Beauty Up:

... the words and concepts [specialists in beauty language] create and use are integral to its construction. They are selling not only cosmetics or body improving products but also hope and fantasy.

(2006, 194)

With this thought in mind, this study has provided some insights into HS naming practices and cross-cultural variations within product names. It is hoped that this investigation will lead to other research inquiries to address questions such as whether function-denoting terms (or lack thereof) influence potential users' perception and buying decisions. This and other inquiries are left for future research.

\section{Note}

I. For clarity and stylistic purposes, lowercase letters are reserved for italicized Chinese terms and common plant names in English,

but capital letters are used for the actual soap names in English.

\section{Disclosure statement}

No potential conflict of interest was reported by the author.

\section{Bibliography}

Charmasson, H. I988. The Name is the Game-How to Name a Company or Product. Homewood, IL: Erwin Professional. Accessed I2 March 20I8. https://www.jadeinstitute.com/jade/oriental-herb-gallery. php?order=chinese_name

Chen, L. 2019. "Communicating Health and Wellness: The Meaning behind Names of Handmade Soaps." English Today 35, no.I: I4-I9.

Cross, M. 20I 5. The Handmade Soap Book, Updated 2nd Edition. Grantham, UK: IMM Lifestyle.

Danesi, M. 20II. "What's in a Brand Name? A Note on the Onomastics of Brand Naming." Names 59, no. 3: $175-85$.

Dong, L., and M. Helms. 200I. "Brand Name Translation Model: A Case Analysis of US Brands in China." Journal of Brand Management 9, no. 2: 99-I I 5.

Fox, K. 2005. The Smell Report. Oxford: Social Issues Research Center. Accessed 8 April 20I8. http:// www.sirc.org/publik/smell.pdf.

Haig, M. 20I I. Brand Failures: The Truth about the roo Biggest Branding Mistakes of All Time. London: Kogan Page.

Hall, M. 2008. "The Marketing of Organic Products: An Instrumental/Symbolic Perspective." Journal of Food Products Marketing I4, no. 3: I-II. 
Haugen, J. 2009. "Borrowed Borrowings: Nahuatl Loan Words in English." E-Journal in Lexicology. 3: 64-I06. http://journals.openedition.org/lexis/638

Hsu, E., and S. Harris. 2010. Plants, Health and Healing: On the Interface of Ethnobotany and Medical Anthropology. New York: Berghahn Books.

Kakudidi, E. 2004. "Cultural and Social Uses of Plants from and around Kibale National Park, Western Uganda." African Journal of Ecology 42, no. si: I I 4-8.

Miller, L. 2006. Beauty Up: Exploring Contemporary Japanese Body Aesthetics. Berkeley: University of California Press.

Nuessel, F. 201 8. “A Note on Selected Craft Beer Brand Names.” Names 66, no. 2: I06-I 5.

Ottman, J. (ed.). 20II. "The New Rules of Green Marketing: Strategies, Tools, and Inspiration for Sustainable Branding." San Francisco: Berrett-Koehler Publishers.

Parsonage Soap Company. 20I 5. "Essential Oil Soaps-Lavender Lime.” Accessed 20 January 20I 8. https:// squareup.com/market/parsonagesoaps/item/lavender-lime

Pérez Hernández, L. 2013. "A Pragmatic-Cognitive Approach to Brand Names: A Case Study of Rioja Wine Brands." Names 6I, no. I: 33-46.

Rondilla, J., and P. Spickard. 2007. Is Lighter Better?: Skin-Tone Discrimination among Asian Americans. Lanham, MD: Rowman \& Littlefield Publishing.

Sarkar, A. N. 20I2. "Green Branding and Eco-Innovations for Evolving a Sustainable Green Marketing Strategy." Asia-Pacific Journal of Management Research and Innovation 8, no. I: 39-58.

APPENDIX 1

NAMES OF PLANTS WITH BICULTURAL APPEAL

\begin{tabular}{lcclcc}
\hline Common Name & Freq/US & Freq/TW & Common Name & Freq/US & Freq/TW \\
\hline Almond & 6 & 3 & Lavender & 14 & 6 \\
Aloe & 3 & 4 & Lemon/Lime & 15 & 6 \\
Apple & 3 & 1 & Lemongrass & 3 & 4 \\
Apricot/Nectarine/Peach & 3 & 4 & Loofah & 2 & 1 \\
Avocado & 1 & 12 & Magnolia & 2 & 1 \\
Calendula & 1 & 16 & Melon & 1 & 6 \\
Carrot & 2 & 1 & Mint & 11 & 6 \\
Cedar & 4 & 6 & Oat & 2 & 7 \\
Chamomile & 1 & 4 & Olive & 4 & 9 \\
Citrus/Orange & 7 & 8 & Pine & 3 & 1 \\
Coconut & 3 & 1 & Rose & 8 & 7 \\
Cucumber & 2 & 2 & Rosewood & 1 & 1 \\
Ginger & 6 & 4 & Rosemary & 6 & 1 \\
Grape/Grapeseed & 1 & 1 & Sandalwood & 3 & 1 \\
Honey & 15 & 14 & Tea Tree & 6 & 7 \\
Honeysuckle & 2 & 2 & Thyme & 1 & 1 \\
Jasmine & 2 & 2 & Verbena & 3 & 1 \\
\hline
\end{tabular}


APPENDIX 2

NAMES OF TCM HERBS

\begin{tabular}{|c|c|c|c|}
\hline $\begin{array}{l}\text { Chinese Name } \\
\text { (Pinyin) }\end{array}$ & $\begin{array}{l}\text { Scientific Name/ } \\
\text { English Translation }\end{array}$ & $\begin{array}{l}\text { Chinese Name } \\
\text { (Pinyin) }\end{array}$ & $\begin{array}{l}\text { Scientific Name/ } \\
\text { English Translation }\end{array}$ \\
\hline ming ri ye & Angelica keiskei & yi mu & Motherwort \\
\hline dong gui & Angelica sinensis & ai cao & Mugwort \\
\hline wei feng cao & Bidens pilosa & lu dou & Mung Bean \\
\hline hui ren & Pearl Barley & gian ma ye & Nettle Leaf \\
\hline zhou shou xiang & Coleus amboinicus & wu ye song & Pinus formosana \\
\hline huang lian & Coptis chinensis & he shou wu & $\begin{array}{l}\text { Polygonum } \\
\text { multiflorum }\end{array}$ \\
\hline mou cao & Desmodium caudatum & zi cao & Purple Gromwell \\
\hline jie gu mu hua & Elderflower & bai he cao & Rhinacanthus nasutus \\
\hline hu lu ba zi & Fenugreek Seed & mei gui guo & Rosehip \\
\hline wu tong & Firmiana simplex & hong ju & Red Yeast Rice \\
\hline jiang geng & Ginger Root & luo shen & Roselle \\
\hline ye jiang huang & Hedychium coronarium & wu haun zi & Soapberry \\
\hline yu xing cao & Houttuynia cordata & jing ying & Tithonia \\
\hline niu xi cao & Hyssop & zi dan & Tournefortia montana \\
\hline ma yin dan & Lantana & yan lan cao & Vetiver \\
\hline
\end{tabular}

\section{Notes on contributor}

Lindsey N. H. Chen is an Associate Professor of General Linguistics at the National Taiwan Normal University. Her areas of interest are language and culture, onomastics, and semantics.

Correspondence to: Dr Lindsey N. H. Chen, National Taiwain Normal University, Taipei City, Taiwan. Email: chenlindsey@yahoo.com 